Proyecciones

Vol. 18, Nº 2, pp. 145-153, December 1999

Universidad Católica del Norte

Antofagasta - Chile

\title{
ON TOPOLOGICAL PROPERTIES OF SKOROKHOD'S INTEGRAL *
}

\author{
EUGENIO SAAVEDRA \\ Universidad de Santiago de Chile, Santiago - Chile
}

\begin{abstract}
We study continuity properties of Skorokhod's integral following the definition of such an integral in [4].
\end{abstract}

AMS Classification : primary 60B10, 60G40.

KEY WORDS : Skorokhod's Integral, Continuity.

*This research was supported by the "Cátedra Presidencial en Análisis Estocástico", and by DICYT N 049833SG. 


\section{Notations - Preliminaries}

1. We begin with some notations. The basic probability space is the set of continuous functions $\Omega=C([0,1], \mathbf{R})$ endowed with the uniform topology. Over $\Omega$ we define the canonical projections $W_{t}(w)=w(t)$, and the associated $\sigma$-algebras $\mathbf{F}_{\mathbf{t}}=\sigma\left(\mathbf{W}_{\mathbf{s}} ; \mathbf{s} \leq \mathbf{t}\right)(t \in[0,1])$. Let $\mathbf{F}$ denote the Borel $\sigma$-algebra of $\Omega$, and let $\mathbf{P}$ denote the Wiener measure on $(\Omega, \mathbf{F})$ with which the process $W$ becomes a Brownian motion.

It is well known that the space $L^{2}(\Omega, \mathbf{F}, \mathbf{P})$ may be descomposed into a direct sum of symmetrical tensor products group $\oplus H^{n \odot}$, where $H$ is the $n \geq 0$

Hilbert space defined as the $L^{2}$-closure of the vector space spanned by finite linear combinations $\sum_{i=1}^{N} a_{i} W_{t_{i}}$. This is the so-called chaos expansion of $L^{2}$.

The chaotic representation leads to the consideration of the following special class of random variables. First we let $C_{p}^{\infty}\left(\mathbf{R}^{\mathrm{m}}\right)$ denote the set of all infinitely differentiable functions $f: \mathbf{R}^{m} \rightarrow \mathbf{R}$ such that $f$ together with all of its derivatives have polynomial growth order. Next we define the set of smooth random variables $S$ consisting of all $F: \Omega \rightarrow \mathbf{R}$ for which there exist a finite collection $t_{1}, \ldots, t_{m} \in[0,1]$ and $f \in C_{p}^{\infty}\left(\mathbf{R}^{\mathbf{m}}\right)$ such that

$$
F(w)=f\left(W_{t_{1}}(w), \ldots, W_{t_{m}}(w)\right) .
$$

By this chaotic representation we have that $\mathcal{S}$ is dense in $L^{2}(\Omega, \mathbf{F}, \mathbf{P})$.

2. For every $F \in \mathcal{S}$, the derivative $D F$ is defined to be the process $D F=$ $\left(D_{t} F\right)_{t \geq 0}$ given by

$$
D_{t} F=\sum_{i=1}^{m} \frac{\partial f}{\partial x_{i}}\left(W_{t_{1}}, \ldots, W_{t_{m}}\right) \cdot I_{\left[0, t_{i}\right]}(t)
$$

where $I_{\left[0, t_{i}\right]}$ stands for the indicator function of the interval $\left[0, t_{i}\right]$, with $t \in[0,1]$.

3. For every $u \in L^{2}([0,1] \times \Omega)$, we define

$$
N(u)=\inf \left\{c>0:\left|\mathbf{E}\left(\int_{0}^{1} D_{t} F u_{t} d t\right)\right| \leq c\|F\|_{L^{2}(\Omega)} ; F \in \mathcal{S}\right\}
$$

where $\|\cdot\|_{p}$ is the norm in $L^{p}(\Omega, \mathbf{F}, \mathbf{P})$. We adopt the convention inf $\phi=\infty$. 
Now we introduce the domain of Skorokhod's integral

$$
\operatorname{Dom}\left(D^{*}\right)=\left\{u \in L^{2}([0,1] \times \Omega): N(u)<\infty\right\} .
$$

The Skorokhod integral of $u \in \operatorname{Dom}\left(D^{*}\right)$ is thon characterized as the unique element $D^{*} u$ in $L^{2}(\Omega)$ such that

$$
\mathbf{E}\left(F D^{*} u\right)=\mathbf{E}\left(\int_{0}^{1} D_{t} F u_{t} d t\right)
$$

for all $F \in \mathcal{S}$.

4. We next introduce a weak topology which will be associated to the construction of Skorokhod's integral. Given a sequence $\left\{Z_{n} ; n \geq 1\right\}$ in $L^{2}(\Omega)$, we say it converges weakly along $\mathcal{S}$ towards a limit $Z_{\infty}$, and write $Z_{n} \stackrel{\mathcal{W}(\mathcal{S})}{\longrightarrow} Z_{\infty}$, if $\mathbf{E}\left(F Z_{n}\right)$ goes to $\mathbf{E}\left(F Z_{\infty}\right)$, for all $F \in \mathcal{S}$.

It is worth noting that if $Z_{n} \stackrel{W(S)}{\longrightarrow} Z_{\infty}$ and $\sup _{n}\left\|Z_{n}\right\|_{L^{2}(\Omega)}<\infty$, then $\left\{Z_{n} ; n \geq 1\right\}$ converges weakly to $Z_{\infty}$ in $L^{2}(\Omega)$. Thus the $\mathbf{W}(\mathcal{S})$-topology is weaker than the usual weak topology $\mathrm{w}$ in $L^{2}(\Omega)$.

5. Let $\mathbf{D}$ denote the $\mathbf{D}$ closure of $\mathcal{S}$ according to the norm $\|\cdot\|_{1,2}$ where

$$
\|F\|_{1,2}=\|F\|_{L^{2}(\Omega)}+\|D \cdot F\|_{L^{2}([0,1] \times \Omega)} .
$$

We say a sequence $\left\{Z_{n} ; n \geq 1\right\}$ in $L^{2}(\Omega)$ is $\mathcal{W}(\mathcal{S})$-weakly uniformly continuous at zero if

$$
\lim _{\|F\|_{1,2} \downarrow 0_{n \geq 1}} \sup \left|\mathbf{E}\left(F Z_{n}\right)\right|=0 .
$$

On the other hand, we will say the sequence $\left\{Z_{n} ; n \geq 1\right\} \subset L^{2}$ is $\mathcal{W}(\mathcal{S})$ weakly bounded if

$$
\sup _{n \geq 1}\left|\mathbf{E}\left(F Z_{n}\right)\right|<\infty
$$

for all $F$ in $\mathcal{D}$. 


\section{Main Results}

1. Proposition. The function $N$ is a norm over $\operatorname{Dom}\left(D^{*}\right)$. The topology defined by $N$ is called the natural topology in $\operatorname{Dom}\left(D^{*}\right)$.

Proof : It is clear that $N$ is a seminorm. Hence if $N(u)=0$, then $\mathbf{E}\left(\int_{0}^{1} D_{t} F u_{t} d t\right)=0$. But this means

$$
\mathbf{E}\left(\int_{0}^{1}\left(\sum_{i=1}^{m} \frac{\partial f}{\partial x_{i}}\left(W_{t_{1}}, \ldots, W_{t_{m}}\right) I_{\left[0, t_{i}\right]}(t) u_{t}\right) d t\right)=0
$$

where $t_{1}, \ldots, t_{m}$ vary over $[0,1]$ and $f$ runs over $C_{p}^{\infty}\left(\mathbf{R}^{\mathrm{m}}\right)$. In particular, letting $f$ vary over the set of polynomials, we have

$$
\mathbf{E}\left(\int_{0}^{1} \sum_{i=1}^{m} \varphi_{i} \otimes \psi_{i}(w, t) u_{t}(w) d t\right)=0
$$

where $\varphi_{i}(w)=P\left(W_{t_{1}}(w), \ldots, W_{t_{m}}(w)\right)$ runs over polynomial functions and $\psi_{i}(t)=I_{\left[0, t_{i}\right]}(t)$. Since the $\varphi_{i}$ 's are total in $L^{2}(\Omega)$ (the Wiener chaos property), and since the $\psi_{i}$ 's have the same property in $L^{2}([0,1])$, the tensor product of both lead to a total family in $L^{2}([0,1] \times \Omega)$. Therefore $(2.2)$ implies $u=0$ almost surely with respect to the product measure $d t d \mathbf{P}$ on $[0,1] \times \Omega$

We now prove some results with relation to the convergence of Skorokhod's integral.

2. Theorem. Let $\operatorname{Dom}\left(D^{*}\right)$ be the domain of Skorokhod's integral which we endow with the natural topology. Then the operator $D^{*}$ from $\operatorname{Dom}\left(D^{*}\right)$ into $L^{1}(\Omega, \mathbf{F}, \mathbf{P})$ is continuous. Furthermore, for every $u \in D$ om $\left(D^{*}\right)$, the following inequality holds

$$
\left\|D^{*} u\right\|_{L^{1}(\Omega)} \leq 2 N(u)
$$

Proof : Note that if we prove (2.3), the first statement follows immediately. For the second, let $A^{+}$(resp. $A^{-}$) denote the set of all $w \in \Omega$ such that $D^{*} u(w)>0\left(\right.$ resp. $\left.D^{*} u(w)<0\right)$. Though to define $A^{+}$and $A^{-}$ 
we should choose a representative function in the class $D^{*} u$, to simplify superflous technicalities we indentify sets which are almost surely equal.

Since $\mathcal{S}$ is dense in $L^{2}(\Omega)$, there exists a sequence $\left(F_{n}^{+}\right)$(resp. $\left(F_{n}^{-}\right)$) of smooth random variables which approximates $I_{A^{+}}$(resp. $I_{A^{-}}$) in the $L^{2}-$ norm. Therefore

$$
\begin{aligned}
\mathbf{E}\left(\left|D^{*} u\right|\right) & =\mathbf{E}\left(I_{A}+D^{*} u\right)-\mathbf{E}\left(I_{A}-D^{*} u\right) \\
& =\lim _{n}\left(\mathbf{E}\left(\int_{0}^{1} D_{t} F_{n}^{+} u_{t} d t\right)\right)-\lim _{m}\left(\mathbf{E}\left(\int_{0}^{1} D_{t} F_{m}^{-} u_{t} d t\right)\right) \\
& \leq \lim _{n}\left(\left|\mathbf{E}\left(\int_{0}^{1} D_{t} F_{n}^{+} u_{t} d t\right)\right|+\left|\mathbf{E}\left(\int_{0}^{1} D_{t} F_{n}^{-} u_{t} d t\right)\right|\right) \\
& \leq N(u) \lim _{n}\left(\left\|F_{n}^{+}\right\|_{2}+\left\|F_{n}^{-}\right\|_{2}\right) \\
& \leq 2 N(u)
\end{aligned}
$$

3. Proposition. Let Dom $\left(D^{*}\right)$ be endowed with the natural topology, and let $L^{2}(\Omega)$ be endowed with the $\mathcal{W}(S)$-topology. Then the operator $D^{*}$ from $\operatorname{Dom}\left(D^{*}\right)$ into $L^{2}(\Omega)$ is continuous. Furthermore, given any sequence $\left\{u^{n} ; n \geq 1\right\}$ in $\operatorname{Dom}\left(D^{*}\right)$ such that it converges to $u \in \operatorname{Dom}\left(D^{*}\right)$ in the natural topology and that $\sup _{n}\left\|D^{*}\left(u^{n}\right)\right\|_{L^{2}(\Omega)}<\infty$, we have that $D^{*}\left(u^{n}\right) \stackrel{w}{\longrightarrow} D^{*}(u)$.

Proof : Given $\left\{u^{n} ; n \geq 1\right\} \subset \operatorname{Dom}\left(D^{*}\right)$ such that $u^{n} \stackrel{N}{\longrightarrow} u$ and $F \in \mathcal{S}$ arbitrary, we have

$$
\begin{aligned}
\left|\mathbf{E}\left(F D^{*}\left(u^{n}\right)\right)-\mathbf{E}\left(F D^{*}(u)\right)\right|=\mid & \mathbf{E} \int_{0}^{1} D_{t} F\left(u_{t}^{n}-u_{t}\right) d t \mid \\
& \leq\|F\|_{L^{2}(\Omega)} N\left(u^{n}-u\right)
\end{aligned}
$$

and the result now follows.

Remark that the above result still holds if we consider the $L^{2}(\Omega \times[0,1])$ topology and use the Cauchy-Schwarz inequality.

4. Corollary Let $u$ be a process such that $u I_{[0, t]} \in \operatorname{Dom}\left(D^{*}\right)$. for all $t \in[0,1]$. Then there exists a sequence of semimartingales $\left(X_{n}\right)_{n \geq 1}$ such that $X_{n}(t)$ converges in the $\mathcal{W}(\mathcal{S})$-topology to the integral $\int_{0}^{t} u(s) d W(s)=$ $D^{*}\left(u 1_{[0, t]}\right)$, for all $t \in[0,1]$. 
Proof : Since $u \in L^{2}(\Omega \times[0,1])$, there exists a sequence of processes $u_{n}$ that approaches $u$ in the the $L^{2}$-norm of the form

$$
u_{n}(t)=F_{0} 1_{\{0\}}+\sum_{i=1}^{N_{n}} F_{i}^{n} I_{\left[t_{i}^{n}, t_{i+1}^{n}\right\}}(t)
$$

where the F/s are smooth random variables and $\pi_{n}: 0=t_{0}^{n}<t_{1}^{n}<\ldots<$ $t_{N_{n}}^{n}=1$ is a sequence of partitions of $[0,1]$ such that $\left|\pi_{n}\right|=\sup _{i}\left|t_{i+1}^{n}-t_{i}^{n}\right| \rightarrow$ 0 as $n \rightarrow \infty$.

Each $F_{i}^{n}$ is a function of a Brownian vector of the form $\left(W_{\alpha_{1}^{n, i}, . .,} W_{\alpha_{m(n, i)}^{n, i}}\right)$. Fix $n$ and arrange all the $\alpha$ 's in a single finite ordered sequence, denoted $p(n)$. Set $\mathcal{H}_{n}=\sigma\left(W_{\alpha} ; \alpha \in p(n)\right)$. All the variables $F_{i}^{n}$ are now $\mathcal{H}_{n}$ - measurable as well as their Malliavin derivatives.

Define $\mathcal{F}_{t}^{\prime \prime}=\cap_{s>t}\left(\mathcal{F}_{s} \cup \mathcal{H}_{n}\right)$, for all $t \in[0,1]$. The process $u_{n}$ now becomes predictable with respect to the new filtration $\mathcal{F}_{l}^{n}$. In addition, $\mathrm{K}$. Itô proved that $W$ becomes a semimartingale with respect to the new filtration. According to the anticipative calculus, $u_{n} I_{[0, t]}$ may be integrated in the Skorokhod sense and we have

$$
D^{*}\left(u_{n} 1_{[0, t]}\right)=(u \cdot W)(t)-\int_{0}^{t} D_{s} u_{n}(s) d s
$$

where the dot - is used to denote stochastic integration of the simple $\left(\mathcal{F}_{t}^{2}\right)$-predictable process $u_{n}$ with respect to the semimartingale $W$. Remark that the second integral on the right-hand side of the above equation is adapted to $\left(\mathcal{F}_{t}^{n}\right)$ as well. So that, for each $n$, the process defined as $X_{n}(t)=$ $D^{*}\left(u_{n} I_{[0, t]}\right)$ is an $\left(\mathcal{F}_{t}^{n}\right)$-semimartingale. Since $u_{n}$ converges to $u$ in $L^{2}(\Omega \times$ $[0,1])$, the result follows from the continuity of $D^{*}$ in the $\mathcal{W}(\mathcal{S})$-topology.

Finally we show a characterization of the $\mathcal{W}(\mathcal{S})$-weak relative compactness.

For a sequence $\left\{Z_{n} ; n \geq 1\right\}$ in $L^{2}(\Omega, \mathbf{F}, \mathbf{P})$, we can now prove the following two results.

5. Proposition. The sequence $\left\{Z_{n} ; n \geq 1\right\}$ is $\mathcal{W}(\mathcal{S})$-weakily uniformly continuous at zero if the sequence $\mathbf{E}\left(\mathbf{F} Z_{\mathbf{n}}\right)$ converges as $n$ tends to infinity, for all $F \in \mathbf{D}$.

Proof : Fix $\alpha>0$ and define

$$
L_{j}=\left\{F \in \mathcal{D}: \forall n, m \geq j, \quad\left|\mathbf{E}\left(F\left(Z_{n}-Z_{m}\right)\right)\right| \leq \alpha\right\} .
$$


The sets $L_{j}$ are closed in $\mathcal{D}$ and the hypothesis of the proposition implies that the union $\cup_{j \geq 1} L_{j}$ is equal to $\mathcal{D}$. By Baire's theorem there exists a closed set $L_{j}$ with interior nonempty: that is, that there exist an index $j \geq 1$, a real number $r>0$, and a random variable $F_{0} \in L_{j}$ such that

$$
\left\|G-F_{0}\right\|_{1,2}<r \quad \text { implies : } G \in L_{j}
$$

that is, $\left|\mathbf{E}\left(G\left(Z_{n}-Z_{m}\right)\right)\right| \leq \alpha, n, m \geq j$.

Consequently if $n \geq j$ and $F$ is such that $\|F\|_{1,2}<r$, then

$$
\begin{aligned}
\left|\mathbf{E}\left(F Z_{n}\right)\right| & =\left|\mathbf{E}\left(\left(F+F_{0}\right) Z_{n}\right)-\mathbf{E}\left(F_{0} Z_{n}\right)\right| \\
& \leq\left|\mathbf{E}\left(\left(F+F_{0}\right) Z_{n}\right)-\mathbf{E}\left(\left(F+F_{0}\right) Z_{j}\right)\right| \\
& +\left|\mathbf{E}\left(F Z_{j}\right)\right|+\left|\mathbf{E}\left(F_{0} Z_{n}\right)-\mathbf{E}\left(F_{0} Z_{j}\right)\right| \\
& \leq 2 \alpha+\left|\mathbf{E}\left(F Z_{j}\right)\right| .
\end{aligned}
$$

Therefore

$$
\sup _{n \geq 1,\|F\|_{1,2} \leq r}\left|\mathbf{E}\left(F Z_{n}\right)\right| \leq 2 \alpha+\sup _{n \leq k \leq j,\|F\|_{1,2} \leq r}\left|\mathbf{E}\left(F Z_{k}\right)\right|
$$

converges to zero as $r$ tends to zero. The proof is now complete.

Using this proposition we can provide necessary and sufficient conditions for a sequence of square integrable randon variables to be weakly relatively compact in the sense of the $\mathcal{W}(\mathcal{S})$-topology.

6. Theorem. A sequence of square integrable random variables

$\left\{Z_{n} ; n \geq 1\right\}$ is $\mathcal{W}(\mathcal{S})$-weakly relatively compact if and only if the following conditions are satisfied:

(i) The sequence $\left\{Z_{n} ; n \geq 1\right\}$ is $\mathcal{W}(\mathcal{S})$-weakly uniformly bounded.

(ii) The sequence $\left\{Z_{n} ; n \geq 1\right\}$ is $\mathcal{W}(\mathcal{S})$-weakly uniformly continuous at zero.

(iii) For any sequence $\left\{Z_{n(k)} ; n \geq 1\right\}$ such that the limit $\lim _{k \rightarrow \infty} \mathbf{E}\left(F Z_{n(k)}\right)$ exits. for all $F \in D$, we have $\left|\lim _{k \longrightarrow \infty} \mathrm{E}\left(F Z_{n(k)}\right)\right| \leq c\|F\|_{2}$.

Proof : We first show that the above three conditions are necessary for the weak $\mathcal{W}(\mathcal{S})$-relative compactness of the sequence $\left\{Z_{n} ; n \geq 1\right\}$. Assume that condition $(i)$ does not hold. In this case we can find a sequence 
$\left\{Z_{n(k)}: k \geq 1\right\}$ such that, for some random variable $F \in \mathcal{D}$, we have that $\left|\mathbf{E}\left(F Z_{n(k)}\right)\right|$ increases to infinity. Then the sequence $\left\{Z_{n(k)}: k \geq 1\right\}$ docs not have any $\mathcal{W}(\mathcal{S})$-weakly convergent subsequence.

If condition (ii) is not truc, any subsequence $\left\{Z_{n(k)} ; k \geq 1\right\}$ is not $\mathcal{W}(\mathcal{S})$-weakly uniformly continuous at zero, and by Proposition 5 it cannot be $\mathcal{W}(S)$-weakly convergent.

Clearly condition (iii) is satisfied, since Definition 1.5 implies that all limit points of the $\mathcal{W}(S)$-weakly convergent subsequence must be square integrable random variables.

Conversely, we now assume that conditions (i) truth (iii) are satisfied. Fix a countable and dense subset $\left\{F_{j} ; j \geq 1\right\}$ on $\mathcal{D}$. Using condition (i) and the classical diagonal argument we can find a subsequence $\left\{Z_{n(k)} ; k \geq 1\right\}$ such that $\mathbf{E}\left(F_{j} Z_{n(k)}\right)$ converges as $k$ tends to infinity, for all $j \geq 1$. The limit, denoted $\phi\left(F_{k}\right)$, is continuous in the $\|\cdot\|_{1,2}$ norm by condition (ii). Hence the sequence $\mathbf{E}\left(F Z_{n(k)}\right)$ converges, for all $F \in \mathcal{D}$, and the limit is continuous in the $L_{2}-$ norn by condition (iii).

\section{References}

[1] G. Bobadilla, R. Rebolledo, E. Saavedra, Sur la convergence d'intégrales anticipatives, Séminaire de Probabilités XXVIII Lect. Notes in Math. , Springer-Verlag, 1583, pp. 113 - 115, (1994).

[2] A.N. Kolmogorov, V.S. Fomine, Elćments de la Théorie des fonctions et de l'Analyse Fonctionnelle, Ed. Mir, Moscou (1974).

[3] G. Da Prato, P. Malliavin, D. Nualart, Compact families of Wiener functionals, C.R. Acad. Sci. Paris, I, 315, pp. 1287 - 1291, (1992).

[4] D. Nualart, E. Pardoux, Stochastic calculus with anticipating integrand, Probability Theory and Related Fields 78, pp. 80 - 129, (1988).

Received: March 02, 1999. 


\section{Eugenio Saavedra}

Departamento de Matemáticas y Ciencias de la Computación Universidad de Santiago de Chile

Casilla 307

Correo 2

Santiago

Chile 